\title{
¿Cuál es el valor de la biopsia aleatoria en el seguimiento del Carcinoma Uroterial Superficial de Vejiga?
}

\author{
W. Eduardo Matheus, R. Nardi Pedro, R.R. Maia Oliveira, U. Ferreira, N. Rodrigues Netto JR
}

Universidad de Campinas - UNICAMP. Brasil.

Actas Urol Esp 2005; 29 (9): 842-845

\begin{abstract}
RESUMEN
¿CUÁL ES EL VALOR DE LA BIOPSIA ALEATORIA EN EL SEGUIMIENTO DEL CARCINOMA UROTERIAL SUPERFICIAL DE VEJIGA?

Introducción: Los pacientes con tumor de vejiga de tipo Carcinoma de Células Transicionales (CCT) presentan enfermedad generalizada de todo el urotelio. El seguimiento de los pacientes con diagnóstico de CCT superficial es sumamente importante por la potencialidad de progresión tumoral. Algunos servicios de urología defienden la biopsia aleatoria de forma rutinaria, sin embargo, otros servicios juzgan ese método innecesario y utilizan la cistoscopia asociada a la citología urinaria como método de seguimiento.

Materiales y Métodos: Fueron analizados, retrospectivamente, 49 pacientes portadores de tumor superficial de vejiga (pTa y pT1), en el periodo de 10 años. El seguimiento de este grupo se realizó a través de exámenes de cistoscopia, trimestrales en el primer año, semestrales en el segundo y tercer año, y anuales en el cuarto y quinto año. Durante las cistoscopias se realizaron análisis de citología de la orina y lavado vesical, biopsias de áreas sospechosas y biopsias aleatórias.

Resultados: En el grupo de biopsias aleatorias (total de 1.489) se observaron 15 biopsias positivas (1\%) y en el grupo de biopsias de áreas sospechosas (total de 38 ) hubo 4 biopsias positivas (10,5\%). Los resultados se consideraron positivos en presencia de tumor urotelial y carcinoma in situ. La citología y/o el lavado vesical se presentaron positivas en 50 $(17,4 \%)$ de las 288 cistoscopias realizadas. La sensibilidad de la biopsia, incluyendo las aleatorias y las dirigidas, fue de $31 \%$, y su especificidad, de $85,2 \%$, mientras que la asociación de la citología y el lavado urinario obtuvieron sensibilidad de $48 \%$ y especificidad de $86,5 \%$.

Conclusión: La biopsia aleatoria no demostró mayor eficiencia en el diagnóstico de recidiva tumoral de CCT superficiales cuando fue comparada a la citología urinaria y el lavado vesical, los cuales presentan buenas tasas de sensibilidad y especificidad.
\end{abstract}

Palabras clave: Tumor de vejiga. Carcinoma de células transicionales. Biopsias aleatorias. Recidiva tumoral. Citología.

\section{ABSTRACT \\ IS THERE A PLACE FOR RANDOMIZED BIOPSIES IN THE FOLLOW UP OF SUPERFICIAL TRANSITIONAL CELL CARCINOMA OF THE URINARY BLADDER?}

Introduction: Transitional cell carcinoma of the bladder represents a disease of entire urothelial tract. The follow up is very important to detect any lesion that might represent a progression or a local recurrence. Some authors recommend randomized biopsies as a routine workup, others recommend cystoscopies and urinary cytology as the main part of superficial bladder cancer follow up.

Patients and Methods: Forty nine patients with superficial bladder cancer were followed up during a ten-year period. Randomized biopsies and urinary cytology were harvested according to the international cancer protocol on bladder cancer.

Results: 15 (1\%) out of 1.489 randomized biopsies found to be positive to transitional cell carcinoma. Four out (10.5\%) of 35 biopsies targeted to suspicious areas were positive to transitional cell carcinoma. 50 (17.4\%) out of 288 cystoscopies with urinary cytology found to be positive to transitional cell carcinoma. Sensitivity and Specificity of biopsies (including randomized and targeted) were $31 \%$ and $85.2 \%$ respectively. Sensitivity and specificity of cystoscopies with urinary cytology were $48 \%$ and $86.5 \%$ respectively.

Conclusion: Randomized biopsies did not show to detect more local recurrence or progression when compared to the urinary cytology. Cystoscopies with urinary cytology have good sensitivity and specificity for detection of tumor recurrence during follow up of transitional cell carcinoma.

Keywords: Transitional cell carcinoma. Randomized biopsies. Tumor recurrence. Urinary cytology. 
$\mathrm{L}$ os pacientes con tumor de vejiga de tipo Carcinoma de Células Transicionales (CCT) presentan enfermedad generalizada de todo el urotelio. Como consecuencia, el tumor presenta característica multifocal y altas tasas de recidiva (en torno de 60\%), después del tratamiento exclusivo con resección local o fulguración ${ }^{1-4}$.

El seguimiento de los pacientes con diagnóstico de CCT superficial es sumamente importante por las características descritas arriba y por la potencialidad de progresión tumoral.

El método más eficaz y al mismo tiempo mejor tolerado por el paciente para la detección precoz de la recidiva tumoral todavía es controvertido. Algunos servicios de urología defienden la biopsia aleatoria de forma rutinaria, sin embargo, otros servicios juzgan ese método innecesario y utilizan la cistoscopia asociada a la citología urinaria como método de seguimiento ${ }^{7-12}$.

El objetivo principal de este trabajo fue comparar el resultado positivo de las biopsias aleatorias (áreas normales) y de las áreas sospechosas, ambas durante procedimiento de cistoscopia. El objetivo secundario fue comparar el valor de todas esas biopsias con la citología urinaria y el lavado vesical, en el seguimiento del CCT.

\section{MATERIAL Y MÉTODOS}

Fueron analizados, retrospectivamente, 49 pacientes portadores de tumor superficial de vejiga (pTa y pT1), en el periodo de 10 años. En ese grupo de pacientes la media de edad era de 60 años, con variación de 26 a 78 años. En el momento del diagnóstico, 26 pacientes presentaban CCT Grado I (bajo grado), 19 pacientes presentaban CCT Grado II y 4 pacientes presentaban CCT Grado III, estos dos últimos considerados de alto grado (Tabla 1).

Inicialmente, con la finalidad de diagnóstico y tratamiento, los pacientes fueron sometidos a resección transuretral completa del tumor vesical (RTU). Después de la definición del tipo histológico y estadificación, los pacientes con tumor localizado y superficial fueron tratados con inmunoterapia intravesical. El tratamiento inmunoterápico utilizado fue el BCG (cepa Moreau), siendo administrados $40 \mathrm{mg} / \mathrm{semana}$, por 6 semanas (inducción), y $40 \mathrm{mg} / \mathrm{mes}$, hasta completar 1 año de tratamiento (mantenimiento).
Tabla 1

\begin{tabular}{cc}
\hline $\begin{array}{c}\text { Grado } \\
\text { (diferenciación histológica) }\end{array}$ & $\begin{array}{c}\text { Número } \\
\text { de pacientes }\end{array}$ \\
\hline I & 26 \\
II & 19 \\
III & 4 \\
\hline
\end{tabular}

El seguimiento de este grupo se realizó a través de exámenes de cistoscopia, trimestrales en el primer año, semestrales en el segundo y tercer año, y anuales en el cuarto y quinto año. Durante las cistoscopias se realizaron análisis de citología de orina y lavado vesical, biopsias de áreas sospechosas y biopsias aleatorias en el fondo vesical, trígono, pared lateral derecha e izquierda, incluyendo también la uretra prostática en los hombres.

En total, se realizaron 288 cistoscopias y 1.527 biopsias, siendo 1.489 biopsias aleatorias de áreas con mucosa de aspecto normal y 38 de áreas sospechosas, comprendiendo locales de lesiones polipoides o cualquier otra lesión atípica. También se realizaron 288 análisis de citología urinaria y lavado vesical.

\section{RESULTADOS}

En el grupo de biopsias aleatorias (total de 1.489) se observaron 15 biopsias positivas (1\%) y en el grupo de biopsias de áreas sospechosas (total de 38) hubo 4 biopsias positivas (10,5\%). Los resultados se consideraron positivos en la presencia de tumor urotelial y carcinoma in situ (Tabla 2).

La citología y/o el lavado vesical se presentaron positivas en $50(17,4 \%)$ de las 288 cistoscopias realizadas, siguiendo los mismos criterios histológicos de biopsia positiva y presencia de células con atipias en la citología, que sugieren proceso tumoral maligno.

Tabla 2

Resultados de las biopsias

\begin{tabular}{lcc}
\hline & $\begin{array}{c}\text { Biopsias } \\
\text { aleatorias }\end{array}$ & $\begin{array}{c}\text { Biopsias de las } \\
\text { áreas sospechosas }\end{array}$ \\
\hline $\mathrm{N}=1.489$ & $\mathrm{~N}=38$ \\
Negativas & $1.474(98,9 \%)$ & $34(89,4 \%)$ \\
$\begin{array}{l}\text { Positivas } \\
\text { (tumor/ca in situ) }\end{array}$ & $15(1,1 \%)$ & $4(10,5 \%)$ \\
\hline
\end{tabular}


La sensibilidad de la biopsia, incluyendo las aleatorias y las dirigidas, fue de $31 \%$, y su especificidad, de $85,2 \%$. Mientras que la asociación de la citología y el lavado urinario obtuvieron sensibilidad de $48 \%$ y especificidad de $86,5 \%$.

\section{DISCUSIÓN}

La característica multifocal del CCT vesical, así como la alta tasa de recidiva tumoral, hacen que sea necesario un seguimiento minucioso de los pacientes diagnosticados y tratados de esta entidad; varias formas de seguimiento ya se propusieron, algunos preconizan procedimientos invasivos, como las biopsias aleatorias asociadas a la cistoscopia, otros preconizan métodos no invasivos, como la citología urinaria y el lavado vesical $^{2-5,12-15}$.

En 1974, el National Bladder Cancer Collaborative Group desarrolló un protocolo que recomendaba la realización rutinaria de citología urinaria, lavado vesical, cistoscopia y biopsias del tumor, en áreas preseleccionadas ${ }^{10}$.

El uso de biopsias aleatorias en el diagnóstico y seguimiento de pacientes con cáncer de vejiga es un tema polémico ${ }^{16}$. En un estudio interactivo en Inglaterra, $32 \%$ de la audiencia seleccionada optó por biopsias aleatorias en pacientes con tumor solitario, en el momento de la resección del tumor vesical ${ }^{17}$.

Por otro lado, el estudio de Young y Soloway ${ }^{3}$, más tarde reforzado por el trabajo de $\operatorname{Lotan}^{18} \mathrm{y}$ otro realizado por Molina Burgos ${ }^{19}$, demostró que la citología urinaria es un indicador eficiente de la presencia de atipia urotelial, también más aceptada por los pacientes por ser un método no invasivo y con sensibilidad superior a las biopsias aleatorias.

Actualmente existe un gran dilema en cuanto al beneficio real de la biopsia aleatoria en el seguimiento del CCT, pues presenta baja positividad y poco auxilio en el seguimiento de esos pacientes. Además, no se trata de un procedimiento inocuo, pudiendo ocasionar sangrados, perforación vesical y riesgo de implante de células tumorales en las áreas donde se realizó la biopsia ${ }^{20-22}$.

En este trabajo, se demuestran las pocas ventajas en la utilización de las biopsias aleatorias. Actualmente, utilizamos la cistoscopia, la citolo- gía urinaria y el lavado vesical, en el seguimiento de CCT superficial de vejiga, debido a su eficacia, y por tratarse de una forma más inocua de seguimiento.

\section{CONCLUSIÓN}

La biopsia aleatoria no demostró mayor eficiencia en el diagnóstico de recidiva tumoral de CCT superficiales cuando comparada a la citología urinaria y el lavado vesical, los cuales presentan buenas tasas de sensibilidad y especificidad.

La indicación de biopsia vesical durante cistoscopia, en el seguimiento de los pacientes con tumor superficial de vejiga, deberá ser reservada para las áreas sospechosas de recidiva tumoral.

\section{REFERENCIAS}

1. Özen H, Remzi D, Akdas A, Ruacan S. Biopsy of Apparently Normal Bladder Mucosa in Patients with Bladder Carcinoma and its Prognostic Importance. Int Urol Nephrol 1983;15(4):327-332.

2. Schade RO, Swinney J. The Association of Urothelial Atypism with Neoplasia: Its Importance in Treatment and Prognosis. J Urol 1973;109(4):619-622

3. Young MJ, Soloway MS. Office Evaluation and Management of Bladder Neoplasms. Urol Clin North Am 1998; 25(4):603-611.

4. Soloway MS, Murphy W, Cox C. Serial Multiple-Site Biopsies in Patients with Bladder Cancer. J Urology 1978; 120:57-59.

5. Kiemeney LA, Witjes JA, Heijbroek RP, Koper NP, Verbeek AL, Debruyne FM. and The Members of The Dutch SouthEast Co-Operative Urological.: Should Random Urothelial Biopsies be Taken from Patients with Primaty Superficial Bladder Cancer? A Decision Analysis. Br J Urol 1994; 73(2): 164-171.

6. Cooper TP, Wheelis RF, Correa RJ Jr, Gibbons RP, Mason JT, Cummings KB. Random Mucosal Biopsies in the Evaluation of Patients with Carcinoma of the Bladder. $\mathrm{J}$ Urol 1981 Jul;126(1):46-48.

7. Denis L, Nowe P, Declercq G. Diagnostic Contribution of Bladder Washing and Multiple Biopsies in Bladder Cancers. Eur Urol 1980;(3):137-138.

8. Mora LB, Nicosia SV, Pow-Sang JM, Ku NK, Díaz JI, Lockhart J, Einstein A. Ancillary Techniques in the Followup of Transitional Cell Carcinoma: A Comparison of Cytology, Histology and Deoxyribonucleic Acid Image Analysis Cytometry in 91 Patients. J Urol 1996 Jul; 156(1): 49-54; discussion 54-55.

9. Heney NM. Natural History of Superficial Bladder Cancer. Prognostic features and long-term disease course. Urol Clin North Am 1992 Aug;19(3):429-433. Review.

10. Heney NM, Ahmed S, Flanagan MJ, Frable W, Corder MP, Hafermann MD. Hawkins I R for National Bladder Cancer Collaborative Group A.: Superficial Bladder Cancer: Progression and Recurrence. J. Urol 1983;130:1083-1086. 
11. Vicente Rodriguez J, Chéchile G, Algaba F Amaral Jr J. Value of Random Endoscopic Biopsy in the Diagnosis of Bladder Carcinoma in situ. Eur Urol 1987;13(3):150152.

12. Harving N, Wolf H, Melsen F. Positive Urinary Cytology after Tumor Resection: Na indicator for Concomitant Carcinoma In Situ. J Urol 1988;140:495-497.

13. Wolf H, Hojgaard K. Urothelial Dysplasia Concomitant with Bladder Tumours as a Determinant Factor for Future New Occurrences. Lancet 1983;16:134-136.

14. Huland H, Klöppel G, Otto U, Droese M. The Value of Histologic Grading and Staging, Random Biopsies, Tumor and Bladder Mucosa Blodd Group Antigens, in Predicting Progression of Superficial Bladder Cancer. Eur Urol 1984; 10:28-31.

15. Mufti GR, Singh M. Value of Random Mucosal Biopsies in the Management of Superficial Bladder Cancer. Eur Urol 1992;22:288-293.

16. Macfarlane JP, Ellis BW, Harland SJ. The Management of Superficial Bladder Cancer: na Interactive Seminar. Br J Urol 1996;78:372-378.

17. Lotan Y, Roehrborn CG. Cost-effectiveness of a modified care protocol substituting bladder tumor markers for cystoscopy for the follow up of patients with transitional cell cancer of bladder: a decision analytical approach. J Urol 2002;167(1):75-79.
18. Fernández Gómez JM, Rodríguez Martínez JJ, Escaf Barmadah S, Pérez García J, García J, Casasola Chamorro. Significance of random biopsies of healthy mucosa in superficial bladder tumor. Arch Esp Urol 2000; 53(9):785-797.

19. Skemp NM, Fernandes ET. Routine bladder biopsy after bacille Calmette-Guerin treatment: is it necessary? Urology 2002;59(2):224-226

20. Eleuteri P, Grollino MG, Pomponi D, Guaglianone S, Gallucci M, De Vita R. Bladder transitional cell carcinomas: a comparative study of washing and tumor bioptic samples by DNA flow cytometry and FISH analyses. Eur Urol 2000;37(3):275-80.

21. May F, Treiber U, Hartung R, Schwaibold H. Significance of random bladder biopsies in superficial bladder cancer. Eur Urol 2003;44(1):47-50.

22. Molina Burgos R, Millán Salvador JM, Oltra Soler JS, Jiménez Cruz JF. Microsatellite analysis in exfoliated cells from urinary sediment. Its utility for the detection of bladder cancer. Comparison with urinary cytology. Actas Urol Esp 2003;27(8):618-628.

Dr. R. Nardi Pedro

Leontina Carvalho Siquera, 62 13094-560 Campinas (Sao Paulo - Brasil) e-mail: rnpedro@unicamp.br

(Trabajo recibido el 18 de marzo 2005) 